\title{
Schon ein bisschen Bewegung würde reichen
}

Eine Langzeituntersuchung aus Norwegen belegt, dass sich Diabetiker ihr hohes Herz-Kreislauf-Risiko bereits durch drei Stunden körperliche Aktivität pro Woche senken können. In der Studie wurden die Daten von 53587 Männern und Frauen aus der norwegischen Nord-Trøndelag Health-Studie erhoben und mit dem Sterberegister Norwegens

Moe B, Eilertsen E, Nilsen TIL. Diabetes Care 2012 Nov 16; doi: 10.2337/dc11-2472 [Epub ahead of print] abgeglichen.

Ergebnisse: Bis zum Jahr 2008 waren 1716 Personen an HerzKreislauf-Erkrankungen ver- storben. Im Vergleich zur Referenzgruppe ohne körperliche Aktivität und Diabetes hatten die 121 inaktiven Diabetiker eine adjustierte Hazard Ratio (HR) von 2,81. Die HR bei den Teilnehmern, die mindestens drei Stunden körperliche Freizeitaktivität angegeben hatten, betrug bei Diabetikern 0,89 und bei Nichtdiabetikern 0,78. Die nach körperlicher Aktivität stratifizierten Analysen zeigten schrittweise immer schwächere Assoziationen mit steigender körperlicher Aktivität.

Quelle (3 Texte): www.lifestyle-telegramm.de

\section{Braunes Fettgewebe als Angriffspunkt?}

Das braune Fettgewebe ist an der Thermogenese ohne Kältezittern und an der Regulation des Körpergewichts von Tieren beteiligt. Es handelt sich um flexibles Gewebe, das durch Stimuli aufgebaut und bei Ausbleiben der Stimuli wieder abgebaut wird. Viele Untersuchungen an Nagetieren kamen zu dem Ergebnis, dass diese Tiere möglicherweise deshalb nicht übergewichtig werden, weil sie eine erhöhte Masse an braunem Fettgewebe bzw. eine hohe Zahl an braunen Fettzellen in verschiedenen Ge-

Boss O, Farmer SR. Front

Endocrinol 2012;3:14.

[Epub ahead of print]

\section{weben aufweisen.}

Da auch der Mensch aktives braunes Fettgewebe hat, wurde die Idee neu aufgegriffen, dass das braune Fettgewebe ein therapeutisches Ziel darstellt, mit dessen Hilfe man durch Übergewicht bedingte Stoffwechselerkrankungen bekämpfen könnte.

Ergebnisse: Die Übersichtsarbeit von Boss und Farmer fasst Untersuchungen an Nagern zusammen, die die Einschätzung stützten, dass bei Übergewichtigen die Aktivierung von BAT einen deutlichen therapeutischen Nutzen hätte. Die Autoren gehen davon aus, dass eine Stimulierung von braunen Fettzellen im weißen Fettgewebe dazu beitragen kann, die Energiebilanz zu regulieren und die Insulinresistenz wie auch die übergewichtsbedingte Inflammation im weißen Fettgewebe zu mindern.

\section{Hoher Medienkonsum verleitet zu Softdrinks}

Je mehr Zeit Kinder und Jugendliche vor Fernseher und Computer verbringen, desto stärker ist der Konsum von süßen Softdrinks und herzhaften Snacks. Zu diesen Ergebnissen kam eine Teilstudie des europäischen HELENA-Projekts. Für die Untersuchung wurden Daten aus der Healthy Lifestyle in Europe by Nutrition in Adolescence Cross-sectional Study herangezogen. 2202 Personen nahmen an der Studie teil, davon 45,5\% im Alter von 12,5 bis 17,5 Jahren.

Santaliestra-Pasías AM, Mouratidou $\mathrm{T}$, Verbestel $\mathrm{V}$ et al. Arch Pediatr Adolesc Med. 2012;166(11):1010-20.
Der Konsum von fester und flüssiger Nahrung wurden mit zwei nicht aufeinander folgenden 24-Stunden-Recalls erhoben.

Ergebnisse: Jungen, die am Wochenende mehr als vier Stunden pro Tag mit Fernsehen, Computerspielen oder Surfen im Internet verbrachten, nahmen mit höherer Wahrscheinlichkeit gesüßte Getränke zu sich (Odds Ratios [OR]: 1,83, 1,99 und 1,73) als jene Jungen, die weniger als zwei Stunden pro Tag diesen Tätigkeiten nachgingen. Gleichzeitig sank bei hohem Medienkonsum der Verzehr von Obst (OR: 0,39, 0,37 und 0,39). Für Mädchen, die viel Zeit mit Fernsehen oder Computerspielen verbrachten, galt Ähnliches (OR für den Konsum gesüßter Getränke: 1,89 bzw. 1,57; OR für den Obstverzehr: 0,43 bzw. 0,40). 